\title{
Adaptação do Inventário Parental "Language Use Inventory (LUI)" para crianças entre 18 e 47 meses para o Português Europeu: Estudo Piloto
}

\section{Adaptation of the Parent Report Language Use Inventory for 18- to 47-months-old children to European Portuguese: a pilot Study}

\author{
Cristiana da Silva Guimarães ${ }^{1}$, Anabela Cruz-Santos², Leandro Almeida ${ }^{2}$
}

\begin{abstract}
RESUMO
Objetivo: A aquisição e o desenvolvimento da linguagem resultam da interação da criança com o meio ambiente. As interações sociais cotidianas com as pessoas e a comunicação com outros permitem que a criança adquira linguagem, sendo a pragmática o sistema de regras que suporta o uso comunicativo da linguagem. A identificação e a avaliação de crianças em risco de desenvolverem transtornos de linguagem são cruciais, tendo em vista a intervenção precoce eficaz. Tendo em vista a relevância da pragmática como componente da linguagem e a escassez, em Portugal, de instrumentos de avaliação da linguagem validados para idades precoces, a finalidade deste estudo consistiu na tradução, adaptação e validação do instrumento Language Use Inventory (LUI), para o português europeu. O LUI é um inventário parental que avalia o desenvolvimento da pragmática entre os 18 e os 47 meses. Métodos: Foram adotados todos os procedimentos recomendados pelas diretrizes internacionais sobre a adaptação de testes, culminando em estudo piloto com uma amostra de 120 inventários, respondidos pelos pais/cuidadores de crianças portuguesas da referida faixa etária. Resultados: Os coeficientes de consistência interna (Alfa de Cronbach) para a versão portuguesa do LUI situaram-se em 0,97 para a escala total e entre 0,71 e 0,96 para as subescalas. Conclusão: Os resultados preliminares da adaptação do LUI-Pt para crianças portuguesas sugerem a validade interna dessa escala.
\end{abstract}

Descritores: Avaliação; Testes de Linguagem; Linguagem Infantil; Comunicação; Intervenção precoce

\begin{abstract}
Purpose: Language acquisition and development takes in account the child's interaction with the surrounding environment. Daily social interactions with people and communication with others allow the child to acquire language being pragmatics considered a system of rules that support the communicative use of language. Identification and assessment of children at risk for language disorders are crucial in order to carry out an effective early intervention. This study was carried out taking into account first, the relevance of pragmatics as a component of language, and second the lack of assessment tools in Portugal to assess these abilities. Therefore, the aim of this study consists on the translation, adaptation and validation of the inventory "Language Use Inventory" (LUI), to European Portuguese. The LUI is a standardized parent report measure designed to assess pragmatic language development in children within 18- to 47-month-old. Methods: All procedures recommended by test adaptation guidelines were adopted in this study. A pilot study was carried out with a sample of 120 inventories, answered by the parents/ caregivers of the Portuguese children in the target age groups. Results: Cronbach's alpha, which is a numerical coefficient of reliability obtained for the scale strongly confirm a very good internal consistency for the LUI-Pt with 0.97 for the total scale, and coefficients between 0, 71 - 0, 96 for the subscales. Conclusion: Preliminary results indicate the internal validity of the LUI-Pt for Portuguese children confirming its clinical usefulness as an assessment tool.
\end{abstract}

Keywords: Evaluation; Language Tests; Child Language; Communication; Early Intervention

\footnotetext{
Trabalho realizado na Universidade do Minho, Braga, Portugal.

(1) Programa de Pós-graduação (Doutorado) em Estudos da Criança, Instituto de Educação da Universidade do Minho - CIEd, Braga, Portugal.

(2) Instituto de Educação da Universidade do Minho - CIEd, Braga, Portugal.

Conflito de interesses: Não

Contribuição dos autores: $C S G$ pesquisador principal, elaboração da pesquisa, elaboração do cronograma, levantamento da literatura, coleta e análise dos dados, redação do artigo, submissão e trâmites do artigo; $A C S$ e $L A$ orientadores, elaboração da pesquisa, elaboração do cronograma, análise dos dados, correção da redação do artigo, aprovação da versão final.

Endereço para correspondência: Cristiana da Silva Guimarães. Instituto de Educação da Universidade do Minho, Campus de Gualtar, $4710-057$ Braga, Portugal. E-mail: tf.cristiana.guimaraes@gmail.com

Recebido em: 20/5/2013; Aceito em: 26/8/2013
} 


\section{INTRODUÇÃO}

O conceito de linguagem é tão vasto que podemos defini-lo conforme a perspectiva em que a observamos, a intenção com que a usamos e os contextos em que ocorre. Assim, numa perspectiva holística, podemos definir a linguagem como um sistema de símbolos (sons, palavras e sinais) organizado de forma regular, que permite aos humanos se comunicarem entre $\mathrm{si}^{(1,2)}$.

A aquisição e desenvolvimento da linguagem resultam da interação da criança com o meio ambiente, ou seja, as interações sociais diárias com as pessoas e a comunicação com os outros estão na origem da aquisição de linguagem, pela criança. Tratando-se de uma competência que integra uma combinação complexa de vários componentes e regras fonológicas, sintáticas, morfológicas, semânticas e pragmáticas ${ }^{(1,2)}$, a aquisição e desenvolvimento de linguagem são progressivos.

O ser humano pode utilizar várias formas de comunicação (oral, gestual, escrita ou por símbolos) e, "quando o código de linguagem é utilizado para comunicar, é um fenómeno intrinsecamente social. A pragmática é o estudo do uso da linguagem, sendo que, quando a linguagem é usada para comunicar, é um comportamento social"(3).

Desde o início da década de 90, vários linguistas dedicaram-se ao estudo da linguagem tendo formulado numerosas teorias acerca do seu desenvolvimento. O nosso foco, neste estudo, refere-se ao desenvolvimento da pragmática, entendida como o domínio das regras de uso da língua, isto é, a capacidade de apreensão e utilização dessas regras, visando a adequação ao contexto da comunicação ${ }^{(1,2)}$. Consequentemente, podemos analisar a pragmática como a capacidade de usar a linguagem de modo apropriado e assertivo nas interações sociais com outras pessoas ${ }^{(4)}$.

Nesta linha de pensamento, podemos enunciar que a pragmática é o sistema de regras que sustenta o uso comunicativo da linguagem. Deste modo, a pragmática da comunicação não verbal inclui a expressão facial, o tom de voz, gestos e postura corporal e, esses aspectos extralinguísticos e paralinguísticos, proporcionam o contexto para a interpretação da mensagem verbal e a intenção comunicativa dos interlocutores. A pragmática da comunicação verbal inclui, entre outras, as regras de troca de turnos, iniciativa comunicativa e resposta ao interlocutor, manutenção de um tópico de conversação, com adequação da expressão facial, e o uso apropriado do léxico nos diferentes contextos sociais ${ }^{(5-9)}$.

O desenvolvimento da pragmática inicia-se desde a tenra idade quando, na interação, o bebê e a mãe trocam o olhar e as expressões faciais. Passados poucos meses o bebê passa a realizar turnos de vocalizações, imitações e a responder à expressão facial da mãe. A troca do olhar e a produção de vocalizações marcam o início do uso comunicativo da linguagem e vêm confirmar que a linguagem influencia o comportamento social (comportamento das outras pessoas) ${ }^{(5-9)}$. Em suma, ao longo do seu desenvolvimento, a criança adquire e faz o uso da língua em que está inserida, podendo mesmo utilizar só uma palavra complementada com expressões faciais, com alterações do tom de voz, ou com o gesto de apontar. Posto isto, a pragmática é uma componente da linguagem que, apesar de não estar claramente definida, é a base da interação comunicativa e transversal às componentes restantes.

Ao longo das últimas décadas, são muitos os estudos acerca da linguagem. No entanto, a pesquisa sobre o desenvolvimento da pragmática é mais recente, em comparação com as componentes restantes da linguagem. A percepção de que a pragmática emerge como uma componente transversal às restantes, atesta a relevância deste estudo.

Decorrente dessa curiosidade científica e, face à falta de instrumentos de avaliação das competências pragmáticas na infância, em português, pretendemos, com este projeto, adaptar e validar um inventário para esse fim. Após extensa pesquisa da literatura internacional sobre os instrumentos de avaliação do desenvolvimento da linguagem na infância, especificamente com relação à pragmática, a nossa escolha recaiu sobre o Language Use Inventory: An Assessment for Young Children's Pragmatic Language Development (LUI) ${ }^{(10)}$.

Esse instrumento enquadra-se nos parent report - inventários parentais -, muito valorizados nos últimos anos, acreditando-se que pais e cuidadores da criança podem realizar essa avaliação, pois são eles que interagem com a criança por mais tempo ${ }^{(11)}$. Os parent report apresentam a vantagem de apresentarem resultados baseados no vasto conhecimento e experiência dos pais com relação às competências linguísticas dos seus filhos, numa variedade de contextos naturais e situações diárias. Essa circunstância facilita a avaliação mais abrangente da competência da criança, aumentando a validade da informação recolhida. Em suma, tais registos parentais resultam, geralmente, em uma avaliação mais realista do desempenho linguístico da criança, o que um teste formal não consegue captar ${ }^{(11-19)}$. Com efeito, o LUI é um inventário em que os pais ou cuidadores avaliam a linguagem da criança em idades precoces, dos 18 aos 47 meses, tendo em conta lógica de avaliação e intervenção centradas na família.

Neste artigo, são apresentados os resultados obtidos no estudo piloto elaborado com a finalidade de traduzir e adaptar o inventário Language Use Inventory: An Assessment for Young Children's Pragmatic Language Development (LUI) para o português europeu. $\mathrm{O}$ estudo faz parte de um projeto de investigação que pretende validar o LUI para a população portuguesa e estabelecer parâmetros normativos para rastreio e diagnóstico dos transtornos de linguagem.

\section{MÉTODOS}

\section{Participantes}

Para este estudo foi reunido, aleatoriamente, um grupo de 120 participantes, dos distritos de Braga e Porto, Portugal. Foram 
distribuídos 180 inventários por creches e jardins-de-infância. Dos 180 inventários, 36 não foram devolvidos e 24 foram excluídos, seguindo as orientações do instrumento original.

Foram considerados critérios de inclusão: idade superior a 18 meses e inferior a 47 meses, inclusive; utilização de linguagem oral; exposição a outras línguas, além do Português, superior a $20 \%$ do tempo e uso do Português como segunda língua, em caso de exposição da criança a essa língua há, pelo menos, um ano. Os critérios de exclusão foram: falta de mais de duas respostas nas 10 subescalas; utilização exclusiva de linguagem não verbal; presença de deficiência auditiva; ocorrência da combinação de prematuridade maior que duas semanas e peso ao nascimento inferior a $2,5 \mathrm{~kg}$; diagnóstico comprovado de transtorno do desenvolvimento; diagnóstico comprovado de atraso ou problema de fala ou linguagem e complicações significativas no nascimento, ou outros problemas maiores, geralmente reconhecidos como resultados no transtorno de linguagem ou do desenvolvimento cognitivo.

Os elementos da amostra foram os pais e cuidadores de crianças do género feminino $(40,8 \%)$ e do gênero masculino $(59,2 \%)$. Descrevemos os participantes, considerando o gênero e cinco faixas etárias (Tabela 1).

Tabela 1. Distribuição da amostra $(n=120)$ por grupos

\begin{tabular}{lcccc}
\hline \multirow{2}{*}{ Grupo } & Faixa etária & \multirow{2}{*}{$\begin{array}{c}\text { Distribuição } \\
\text { em meses }\end{array}$} & \multicolumn{2}{c}{ Gênero (\%) } \\
\cline { 4 - 5 } & $18-23$ & 16,7 & 9,2 & 7,5 \\
\hline 1 & $24-29$ & 27,5 & 10 & 17,5 \\
2 & $30-35$ & 24,2 & 8,3 & 15,9 \\
3 & $36-41$ & 20,0 & 7,5 & 12,5 \\
4 & $42-47$ & 11,6 & 5,8 & 5,8 \\
5 & & & & \\
\hline
\end{tabular}

\section{Instrumento}

O instrumento utilizado na realização deste estudo foi o Language Use Inventory: An Assessment for Young Children's Pragmatic Language Development LUI ${ }^{(10)}$, um inventário parental padronizado. O LUI foi desenvolvido com base em um projeto de investigação, realizado no Canadá, durante dez anos, e centrado no desenvolvimento precoce da pragmática em crianças dos 18 aos 47 meses de idade.

Esse inventário é constituído por 14 subescalas, organizadas em três partes: Parte 1: Como é que o seu filho comunica-se através de gestos (duas subescalas); Parte 2: Comunicação do seu filho através de palavras (três subescalas); Parte 3: Frases ditas pelo seu filho (nove subescalas). A pontuação total do LUI é obtida através da soma das pontuações de dez subescalas das partes 2 e 3 (C e D; F - K, M e N). As subescalas E e L não estão incluídas na pontuação total por requererem resposta escrita por parte dos pais/cuidadores, de modo a serem fornecidas informações descritivas adicionais sobre as brincadeiras e os tópicos de conversação privilegiados da criança. As três partes permitem avaliar a comunicação da criança em vasta variedade de configurações e funções comunicativas, como por exemplo: pedir ajuda, partilhar o foco de atenção, perguntar e comentar sobre as coisas e as pessoas, orientar as interações com outras pessoas, partilhar o humor, falar sobre a linguagem e as palavras, adaptar o discurso às outras pessoas e construir frases mais longas e histórias.

O inventário LUI permite a identificação de crianças com atraso ou transtorno do desenvolvimento da componente pragmática da linguagem, ou seja, o uso da linguagem em diversos contextos e interações sociais, em relação a crianças do mesmo gênero e idade (em meses). De acordo com a extensa investigação realizada pela sua autora, o inventário apresenta índices de confiabilidade e validade favoráveis à sua utilização na prática clínica.

\section{Procedimentos}

O processo de tradução e adaptação do inventário foi moroso e complexo, seguindo os parâmetros de outras provas psicológicas similares. A par da tradução e da retroversão, procedemos às análises qualitativas, buscando a relevância e a compreensão dos itens pelos pais/cuidadores. Esse processo contempla vários passos que não podem ser negligenciados, como forma de assegurarmos o rigor e o valor das informações recolhidas ${ }^{(20-25)}$.

\section{Pedido de autorização}

Independentemente do tipo de instrumento que se pretende traduzir, há um conjunto de questões legais relacionadas aos direitos de autor e que devem ser salvaguardadas ${ }^{(21)}$. Sendo assim, solicitamos a autorização à autora do instrumento e à editora Knowledge in Development, para efeitos da tradução, adaptação e validação do Language Use Inventory: An Assessment for Young Children's Pragmatic Language Development" para a língua portuguesa. Após aprovação, foram emitidas e assinadas as declarações de autorização de tradução do instrumento LUI para propósitos de investigação.

\section{Tradução}

Nesse procedimento, seguimos determinados pressupostos para que houvesse similaridade entre o instrumento original e a respectiva tradução. $\mathrm{O}$ contato com a autora do inventário e a consulta à especialistas assegurou que, na sua versão final, o instrumento traduzido tivesse equivalência em termos de conteúdo e de semântica dos itens ${ }^{(21)}$. Assim, primeiramente, o instrumento LUI foi traduzido do Inglês para o Português, por um nativo português com fluência na língua inglesa. Em seguida, realizou-se a retroversão, ou seja, a versão traduzida para o Português europeu foi novamente traduzida para o inglês, por um nativo inglês com fluência na língua portuguesa, seguindo as normas internacionais para essa finalidade. As duas versões, a original e a traduzida, foram comparadas e verificou-se, na sua generalidade, a manutenção de equivalência técnica, conteúdo, semântica e critério. 


\section{Revisão da tradução e adaptação sociocultural}

Devido às prováveis diferenças socioculturais entre os diferentes países, e, neste caso, entre Canadá e Portugal, verificamos a existência de coerência de todos os itens, em ambas as línguas ${ }^{(21)}$. Para tal, foi constituído um grupo de três peritos da área da linguística e do desenvolvimento da criança em idades precoces, que efetuou a revisão do instrumento e sugeriu algumas alterações semânticas (verbos, adjetivos, nomes comuns) e sintáticas (simplificação dos enunciados), de modo a adaptar-se à população portuguesa. As duas versões, a original e a traduzida, foram comparadas e identificaram-se evidências da sua equivalência em termos de competências avaliadas e adequação cultural aos respetivos contextos.

\section{Reflexão falada (thinking aloud)}

De forma a detectar a existência de itens mal construídos e de ambiguidade de conteúdo dos itens, que pudessem causar dúvidas e que, portanto, necessitassem ser modificados, realizamos a reflexão falada com um pequeno grupo de pais ${ }^{(20)}$, constituído por dez pais provenientes de regiões urbanas ou rurais, com alta densidade populacional. Houve a preocupação de que esses pais fossem naturais do norte e do sul de Portugal, para melhor atenção às singularidades linguísticas. Após a reflexão falada com todos os participantes, procedemos à análise das suas opiniões e observações. Neste sentido, foram efetuadas as seguintes alterações: aspecto e estrutura do inventário - layout do cabeçalho, sublinhados e negritos; aspectos morfossintáticos - simplificação das frases, retirando-se os pronomes pessoais, iniciando-se a frase pela forma verbal e uniformização, em todas as frases, do pronome pessoal referente ao pai/mãe, que passaram para a $3^{a}$ pessoa do singular; aspectos semânticos - adequação dos exemplos à cultura portuguesa. No final, elaboramos a primeira versão do instrumento em língua portuguesa, com a manutenção de todos os itens, em relação à versão original.

\section{Estudo piloto}

O estudo piloto é o procedimento que antecede o estudo normativo para validação do instrumento à população portuguesa e tem como objetivo verificar a dispersão das respostas, a consistência interna dos itens e a validade do próprio instrumento ${ }^{(20-25)}$. Assim, foi reunido um grupo de participantes, constituindo-se uma amostra de 120 pais e cuidadores com as mesmas características da amostra da população alvo, aos quais foi distribuído o LUI-Pt (versão portuguesa). Seguindo as normas do Código da Comissão de Ética da Universidade do Minho, os participantes assinaram o Termo de Consentimento Informado, sendo-lhes assegurada a confidencialidade das respostas. Os protocolos foram analisados estatisticamente, através do programa SPSS, versão 20.0.

\section{RESULTADOS}

A primeira versão traduzida do LUI contou com 180 itens, correspondendo, assim, aos itens da versão original. Na escala, a maioria dos itens (89\%) corresponde a respostas dicotômicas, do tipo "sim" ou "não" e os itens restantes (11\%) assumem respostas num formato tipo likert, com as opções "já não usa" (apenas presente na subescala A) "nunca", "raramente", "às vezes", ou "frequentemente".

Além do preenchimento do LUI, os pais forneceram informação relativas ao nascimento, saúde e linguagem de seus filhos, por meio de um formulário preparado para esse fim, em que informaram, também, se a criança estava exposta a outra língua, além da língua portuguesa.

Para a verificação do índice de consistência interna do instrumento, foi calculado o valor de Alfa Cronbach para as três partes e as 14 subescalas do LUI, na sua versão adaptada à língua portuguesa (Alpha PT). Os coeficientes obtidos, comparados aos da versão original (Alpha EN) ${ }^{(19)}$, estão descritos na Tabela 2.

Face aos índices obtidos, as três partes do LUI-Pt apresentaram alta consistência interna $(\alpha>0,98)$. Contudo, analisando cada subescala, separadamente, concluiu-se que as subescalas A, C, G, H, I, K, M e N tiveram elevada consistência interna ( $\alpha$ entre 0,84 e 0,96 ), as subescalas $\mathrm{D}, \mathrm{Fe} \mathrm{J}$, um índice adequado de consistência interna ( $\alpha$ entre 0,7 e 0,8 ) e a subescala $B$, fraca consistência interna dos seus itens $(\alpha<0,3)$. As subescalas $\mathrm{E}$ e L são de resposta aberta e como não apresentam pontuação numérica, não foram incluídas na pontuação final do LUI-Pt.

De modo a verificar as diferenças e a aplicabilidade do instrumento, dividimos a amostra em dois subgrupos. O subgrupo 1, formado pelos participantes dos 18 aos 30 meses e o subgrupo 2, pelos participantes dos 31 aos 47 meses, apresentando-se os resultados na Tabela 3.

\section{DISCUSSÃO}

O primeiro aspecto a salientar neste estudo é o tempo de preenchimento do LUI. Na versão original, a autora menciona que os pais/cuidadores levaram entre 22 a 35 minutos para preencherem o LUI ${ }^{(19)}$. Em Portugal, no preenchimento da versão LUI-Pt, constatamos que os pais/cuidadores portugueses utilizaram entre 30 e 45 minutos.

Na sua versão original, segundo O'Neill, o LUI apresenta valores do coeficiente alfa para as três partes e para sete das 12 subescalas de 0,9 ou superior. $O$ valor de alfa para as partes 2 e 3, que compreende o valor total da pontuação do LUI, foi de 0,99 . Os valores de alfa para as subescalas remanescentes, exceto a subescala $\mathrm{B}$, foram todos de níveis aceitáveis, ou acima de 0,80 . O baixo valor de alfa para a subescala $\mathrm{B}(0,55)$ pode ser explicado pelo reduzido número de itens, comparando-se com as outras subescalas, e pelo fato de todas as faixas etárias estarem próximas do teto máximo ${ }^{(19)}$.

As três partes do LUI-Pt e as subescalas apresentaram boa confiabilidade, exceto a subescala B, que mostrou fraca consistência interna, o que pode ser justificado pelo fato de, estando 
Tabela 2. Valores para LUI Original e adaptado em Português Europeu

\begin{tabular}{|c|c|c|c|}
\hline & Alfa IN & Alfa PT & $\mathrm{N}^{\circ}$ de itens \\
\hline Parte 1: Como é que o seu filho se comunica através de gestos & 0,91 & 0,87 & 13 \\
\hline A: Como é que o seu filho usa os gestos para perguntar por alguma coisa & 0,92 & 0,88 & 11 \\
\hline B: Como é que o seu filho usa os gestos para lhe comunicar alguma coisa & 0,55 & 0,29 & 2 \\
\hline Parte 2: A comunicação do seu filho através de palavras & 0,95 & 0,92 & 28 \\
\hline C: Tipos de palavras que o seu filho usa & 0,93 & 0,91 & 21 \\
\hline D: Pedidos de ajuda do seu filho & 0,87 & 0,73 & 7 \\
\hline E: Interesses do seu filho & --- & --- & 2 \\
\hline Parte 3: Frases ditas pelo seu filho & 0,99 & 0,98 & 133 \\
\hline F: Como é que o seu filho utiliza as palavras para conseguir a sua atenção & 0,83 & 0,72 & 6 \\
\hline G: Perguntas e comentários do seu filho acerca das coisas & 0,91 & 0,87 & 9 \\
\hline H: Perguntas e comentários do seu filho acerca dele e de outras pessoas & 0,98 & 0,94 & 36 \\
\hline I: O seu filho utiliza palavras em atividades com os outros & 0,94 & 0,92 & 14 \\
\hline J: Provocações e senso de humor do seu filho & 0,80 & 0,71 & 5 \\
\hline $\mathrm{K}$ : Interesse do seu filho por palavras e linguagem & 0,86 & 0,84 & 12 \\
\hline L: Interesses do seu filho quando fala & --- & --- & 4 \\
\hline M: Como é que o seu filho adapta os temas de conversa & 0,93 & 0,92 & 15 \\
\hline $\mathrm{N}$ : Como é que o seu filho constrói frases ou histórias & 0,98 & 0,96 & 36 \\
\hline
\end{tabular}

Legenda: IN = inglês (LUI Original); PT = português (LUI adaptado para Português Europeu)

Tabela 3. Valores do Alfa Cronbach para os Subgrupos 1 e 2

\begin{tabular}{|c|c|c|}
\hline & $\begin{array}{c}\text { Subgrupo } 1 \\
(18-30 \mathrm{~m})\end{array}$ & $\begin{array}{c}\text { Subgrupo } 2 \\
(31-47 \mathrm{~m})\end{array}$ \\
\hline Parte 1: Como é que o seu filho se comunica através de gestos & 0,82 & 0,85 \\
\hline A: Como é que o seu filho usa os gestos para perguntar por alguma coisa & 0,83 & 0,87 \\
\hline B: Como é que o seu filho usa os gestos para the comunicar alguma coisa & * & 0,37 \\
\hline Parte 2: A comunicação do seu filho através de palavras & 0,92 & 0,88 \\
\hline C: Tipos de palavras que o seu filho usa & 0,91 & 0,88 \\
\hline D: Pedidos de ajuda do seu filho & 0,75 & 0,59 \\
\hline E: Interesses do seu filho & --- & --- \\
\hline Parte 3: Frases ditas pelo seu filho & 0,98 & 0,97 \\
\hline F: Como é que o seu filho utiliza as palavras para conseguir a sua atenção & 0,80 & 0,52 \\
\hline G: Perguntas e comentários do seu filho acerca das coisas & 0,83 & 0,72 \\
\hline H: Perguntas e comentários do seu filho acerca dele e de outras pessoas & 0,96 & 0,93 \\
\hline I: O seu filho utiliza palavras em atividades com os outros & 0,90 & 0,88 \\
\hline J: Provocações e senso de humor do seu filho & 0,59 & 0,69 \\
\hline K: Interesse do seu filho por palavras e linguagem & 0,79 & 0,74 \\
\hline L: Interesses do seu filho quando fala & --- & --- \\
\hline M: Como é que o seu filho adapta os temas de conversa & 0,90 & 0,84 \\
\hline $\mathrm{N}$ : Como é que o seu filho constrói frases ou histórias & 0,95 & 0,93 \\
\hline
\end{tabular}

* Não foi possível o cálculo por se tratar apenas de itens onde se verifica efeito do teto

o coeficiente alfa dependente da variância dos resultados nos itens e no total da subescala, o seu cálculo ter sido afetado por se tratar de uma subescala com apenas dois itens. Mesmo assim, a diferença entre o valor obtido e o coeficiente encontrado na versão original, faz-nos pensar em maior heterogeneidade de respostas dos pais aos dois itens, em Portugal, o que merece ser compreendido, podendo, inclusive, justificar a inclusão de mais itens nessa subescala.

Assim, da análise dos valores de alfa das duas versões do inventário, podemos concluir que os coeficientes obtidos na versão traduzida foram similares aos da versão original, apresentando boa confiabilidade. Essa proximidade é clara, se compararmos a pontuação total das partes 2 e 3 para os 161 itens, em que o coeficiente alfa foi de 0,99 para a versão original 
e 0,98 para a versão traduzida e também está presente nos itens individualmente considerados e nas subescalas.

Adicionalmente, foram analisados os coeficientes alfa para os subgrupos 1 (18 a 30 meses) e 2 ( 31 a 47 meses), onde se verificou, na sua maioria, boa confiabilidade das subescalas. Ressalta-se que, para o subgrupo 1 , não foi possível calcular o coeficiente alfa para a subescala B, por se tratar de uma subescala com apenas dois itens e onde se verificou desempenho uniforme em todos os participantes da amostra. No subgrupo 1, a subescala J $(0,59)$ e no Subgrupo 2, as subescalas B $(0,37)$, D $(0,59)$ e F $(0,52)$ apresentaram baixa consistência interna. Esses valores dos coeficientes parecem indicar ausência de variabilidade de respostas que pode ser justificada pelo reduzido número de itens que constituiu as respectivas subescalas, ou elevada frequência de uso do comportamento comunicativo.

\section{CONCLUSÃO}

A relevância da linguagem no processamento cognitivo-linguístico da criança, desde a primeira idade e a escassez de provas que avaliem a pragmática da linguagem, em Portugal, justificam a escolha para a tradução e adaptação do LUI, cuja versão original mostra utilidade clínica/educacional na avaliação de competências das pragmáticas em crianças de língua inglesa.

Assim, de posse da versão portuguesa adaptada (LUI-Pt), avançamos para um estudo das suas propriedades métricas, registando-se índices de consistência interna muito similares aos valores obtidos com a versão original. Apenas uma subescala apresenta coeficiente alfa bastante reduzido, o que nos parece explicável pelo fato de ser constituída por apenas dois itens. Mesmo assim, justifica-se análise complementar, pois indica que os pais portugueses não associam o mesmo padrão de resposta aos dois itens, podendo-se, inclusive, pensar no reforço do número de itens nessa subescala.

Concluída a fase de adaptação do inventário para a língua portuguesa, iniciaremos o estudo de validação e aferição (normas), tendo em vista obter critérios de desempenho e marcadores linguísticos referentes às competências pragmáticas, em crianças dos 18 aos 47 meses.

No final de todo esse processo, espera-se que o LUI-Pt venha a ser uma ferramenta de avaliação utilizada para intervenção precoce já na primeira infância, por ser capaz de avaliar as competências pragmáticas em idades tão precoces e, deste modo, possibilitar o diagnóstico e intervenção precoce em crianças com transtornos de linguagem e/ou distúrbios de comunicação.

\section{AGRADECIMENTOS}

À Fundação da Ciência e Tecnologia, I.P, pela bolsa de doutoramento, apoio concedido para realização dessa pesquisa, sob processo número SFRH/BD/76861/2011.

\section{REFERÊNCIAS}

1. Sim-Sim. Desenvolvimento da linguagem. Lisboa: Universidade Aberta; 1998.

2. Guidetti M, Nicoladis E. Introduction to special issue: gestures and communicative development. First Language. 2008;28(2):107-15.

3. Gallagher T, Prutting CA. Pragmatic assessment and intervention issues in language. San Diego: College-Hill Press; 1983.

4. Bates E. Language and context: the acquisition of pragmatics. New York: Academic Press; 1976.

5. Dunst CJ. Revisiting "rethinking early intervention". Topics in Early Childhood Special Education. 2000;20(2):95-104.

6. Owens R. Language development: an introduction. 6th ed. Boston: Pearson Education; 2005.

7. Dale PS. Is early pragmatic development measurable? J Child Lang. 1980;7(1):1-12.

8. Iverson JM, Goldin-Meadow S. Gesture paves the way for language development. Psychol Sci. 2005;16(5):367-71.

9. O'Neill DK. The language use inventory for young children: a parent-report measure of pragmatic language development for 18- to 47-month-old children. J Speech Lang Hear Res. 2007;50(1):214-28.

10. O’Neill D. Language use inventory: an assessment for young children's pragmatic language. Canada: Knowledge in Development; 2009.

11. Law J, Roy P. Parental report of infant language skills: a review of the development and application of the communicative development inventories. Child and Adolescent Mental Health. 2008;13(4):198206.

12. Dale PS, Bates E, Reznick JS, Morisset C. The validity of a parent report instrument of child language at twenty months. J Child Lang. 1989;16(2):239-49.

13. Rescorla L. The language development survey: a screening tool for delayed language in toddlers. J Speech Hear Disord. 1989;54(4):58799.

14. Camaioni L, Castelli MC, Longobardi E, Volterra V. A parent report instrument for early language assessment. First Language. 1991;11(33):345-59.

15. Plante E, Vance R. Selection of preschool language tests: a databased approach. Lang Speech Hear Serv Sch. 1994;25(1):15-24.

16. Girolametto L. Development of a parent report measure for profiling the conversational skills of preschool children. Am J Speech Lang Pathol. 1997;6(4):25-33.

17. Squires JK, Potter L, Bricker DD, Lamorey S. Parent-completed developmental questionnaires: effectiveness with low and middle income parents. Early Childhood Research Quarterly. 1998;13(2):345-54.

18. Thai DJ, O'Hanlon L, Clemmons M, Fralin L. Validity of a parent report measure of vocabular and syntax for preschool children with language impairment. J Speech Lang Hear Res. 1999;42(2):482-96.

19. Boudreau D. Use of a parent questionnaire in emergent and early literacy assessment of preschool children. Lang Speech Hear Serv Sch. 2005;36(1):33-47

20. Almeida L, Freire T. Metodologia da investigação em psicologia da 
educação. 5a ed. Braga: Psiquilíbrios; 2008.

21. Geisinger K. Cross-cultural normative assessment: translation and adaptation issues influencing the normative interpretjation of assement instruments. Psychol Assess. 1994;6(4):304-12.

22. Bishop DV. Development of the children's communication checklist (CCC): a method for assessing qualitative aspects of communicative impairment in children. J Child Psychol Psychiatry. 1998;39(6):87991.

23. Allalouf A, Hambleton R, Stephen G. Identifying the causes of DIF in translated verbal Items. Journal of Educational Measurement. 1999;36(3):185-98.

24. Feldman H, Dale PS, Campbell TF, Colborn DK, Kurs-Lasky M, Rockette HE, et al. Concurrent and predictive validity of parent reports of child language at ages 2 and 3 years. Child Dev. 2005;76(4):856-68.

25. Paul D, Roth FP. Guiding principles and clinical applications for speech-Language pathology practice in early intervention. Lang Speech Hear Serv Sch. 2011;42(3):320-30. 